\title{
Age-Related Changes in the Melatonin and Thymulin Biorhythms as Risk Factors for Human Neurodegenerative Diseases
}

\author{
Irina F Labunets* \\ Department of Cell and Tissue Technologies, Institute of Genetic and Regenerative Medicine National Academy of Medical Sciences of Ukraine, Ukraine \\ *Corresponding author: Irina Labunets, Experimental Modeling Laboratory, Cell and Tissue Technologies Department, Institute of Genetic and \\ Regenerative Medicine National Academy of Medical Sciences of Ukraine, Vyshgorodskaya Street 67, PO Box: 04114, Kiev, Ukraine
}

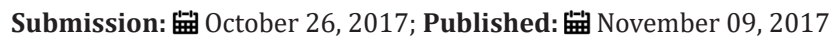

\begin{abstract}
The frequency of age-related neurodegenerative pathology, such as Parkinson's and Alzheimer's diseases, brain ischemia and multiple sclerosis increases worldwide and has great socio-economic implications. The use of approaches based on investigation the pathogenic factors of their development may be perspective in the prevention and treatment of such diseases.

Circadian and circannual rhythms play a key role in the adaptative changes of human neuroendocrine and immune systems to the light and temperature. The rhythmicity of these systems is disturbed in human aging that may be connected with desynchronosis of pineal gland melatonin production. This process becomes more intensive during development of the neurodegenerative diseases.

Thymic endocrine function (thymulin production) is the important part of chronobiological organization of immune system. The circadian and circannual rhythms of thymulin depend on the synchronizing influence of melatonin. Melatonin production disorders lead to the intra-immune desynchronosis via disturbances of the circadian and circannual rhythms of thymulin and its interactions with the endocrine glands (hypophysis, adrenal glands, gonads etc) both in aging and age pathology.

Melatonin treatment improves sleep-wake rhythm and produces antiapoptotic, antioxidant and anti-inflammatory effects in the brain at neurodegenerative diseases and slows down development of this pathology. Moreover, melatonin treatment leads to the suppression of age-related changes in the rhythmicity of immune and neuroendocrine systems functions. Such melatonin effect is realized via synchronous influence on the circadian and circannual rhythms of thymulin production.
\end{abstract}

Therefore melatonin seems to be perspective for restoring disturbed rhythmicity of immune and neuroendocrine systems in:

A. accelerated human aging with pineal gland dysfunction and

B. Patients of different age and sex suffering from neurodegenerative diseases making individual hormone dose correction.

Keywords: Melatonin; Thymulin; Rhythms; Age; Neurodegenerative pathology

\section{Introduction}

Circadian and circannual rhythms play a key role in human organism adaptation to such fluctuating environmental factors as light and temperature [1]. In aging there occurs dyscoordination of rhythms and disturbance of adaptive capacity of many organism functions, in particular of the neuroendocrine and immune systems $[2,3]$. Of a special interest is the regulator of biorhythms of the pineal gland hormone, melatonin, and the regulator of immune function of the thymic hormone, thymulin [4,5]. Many investigators have revealed age-related changes in the functions of pineal gland and thymus and their impact on lifespan in humans [6,7]. Dysfunctions of the pineal gland and thymus coincide with the development of age-dependent pathology.
This article reviews the published data about circadian and circannual rhythms of the melatonin and thymulin in adult and elderly human subjects and their link with disturbances in the rhythmicity of neuroendocrine and immune system functions both during aging and neurodegenerative diseases.

Rhythmicity of Fluctuations in Melatonin and Thymulin Levels in Adult Human Organism and its Alteration in Aging

\section{Melatonin}

Numerous studies suggest that melatonin is the main regulator of circadian and circannual rhythms both in mammals and humans 
$[4,6]$. It closely functions with the endogenic generator rhythms - suprachiasmatic nucleus (SCN) of the hypothalamus. In young subjects melatonin production during dark period and with shortening season photoperiod is increased.

Age-dependent changes in the circadian rhythm of melatonin production and their possible mechanisms were described in many publications. In particular, the nocturnal peak of blood melatonin was found to be lower in the majority elderly and old people versus young subjects [6,8-10]. In our study we also registered disturbances in the circannual blood melatonin rhythms during human aging: the winter hormone peak was decreased or shifted to the spring period [11]. Korkusko and co-workers [8] found the link between saved circadian rhythmicity of blood melatonin in old people and organism adaption and lifespan.

Melatonin influence is largely realized via functioning of the hypothalamus-pituitary-peripheral endocrine glands axes $[6,10]$. The functional state and circadian rhythmicity of organs of these axes are changed in aging but improved after melatonin administration $[6,10]$. According to our data, there is also a link between age-related changes in the circannual rhythms of blood melatonin, adrenocorticotrophic hormone (ACTH) and cortisol levels in human subjects [11]. Interestingly, we observed seasonal rhythm disturbances of melatonin level as early as at 40 years and outstrip desynchronization of ACTH and cortisol levels.

So, the pineal gland dysfunction in aging may be the pathogenic factor of age-related desynchronosis of the neuroendocrine system, and, as a result, promote development of age-dependent disturbances in neurogenesis. Thus, in adult brain there occurs circadian rhythmicity in the proliferation of neural stem cells (NSCs) [12] and olygodendrocyte functioning [13]. Melatonin enhances proliferation and differentiation of NSCs in the subventricular zone of the hypothalamus via stimulation of expression of brain growth factors [14]. Data about influence of sex and glucocorticoid hormones on the brain neurogenesis were reported $[15,16]$.

\section{Thymulin}

Immune system functions are also under control of the pineal gland [17]. According to our data and data of other investigators, the circadian and circannual rhythms in healthy young human subjects were observed in the peripheral blood contents of different $\mathrm{T}$ cell subpopulations, B- and natural killer (NK) cells, granulocytes, and blood immunoglobulin (Ig) and some cytokine levels [18-21].

At the same time, the functioning of immune system is under regulatory influence of its central organ-the thymus [7]. As has been described in the review of Csaba [5], the thymus is not only the lymphoid organ. It also serves as the endocrine organ producing the following hormones: thymic serum factor (FTS) or thymulin, thymosine, thymopoetin and thymus humoral factor. The highly active thymulin demonstrates the biological properties of all known thymic hormones $[22,23]$. In particular, thymulin influences on the differentiation of different thymocyte subpopulations, on the balance of regulatory $\mathrm{T}$ lymphocytes, proliferation and migration
$\mathrm{T}$ cells, etc. Importantly, age-related changes of thymic function precede immune system changes in aging [7].

The data of literature and our own data have shown the similar nocturnal peaks of blood thymulin and melatonin levels in young human subjects $[10,24]$. Besides, we revealed seasonal similarities in the rhythmicity of these hormones in young subjects and their changes in aging [11]. Thus, in aging the circadian rhythm of thymulin is associated with less intensive rise of its nocturnal level while circannual thymulin rhythm shows the shift of its seasonal acrophase from autumn to spring [11]. It is characteristic of old human individuals that age changes of circannual rhythms of immunological indices coincide with seasonal desynchronosis of thymulin [11]. Besides, in our data, the thymus of elderly and old subjects can elevate thymulin production under the influence of activating stimules, in particular melatonin [10].

Melatonin produces impact on the thymic epithelial cells and lymphocytes directly via its own receptors or via changes in the production of hormones of the hypophysis, adrenal and thyroid glands and gonads $[25,26]$. Our data demonstrated improvement of the biorhythms of thymulin and cortisol levels owing to melatonin treatment in the elderly subjects with pineal gland dysfunction [10]. In the comparison with our younger patients this effect was registered with lesser melatonin doses.

Thymulin had reverse effects on the hypothalamuspituitary-adrenal and hypothalamus-pituitary-gonad axes [22]. Experimentally we have shown reverse thymulin effects on the rhythmicity of melatonin production by the pineal gland [27]. Efficacy of such thymulin influence was lesser in old organism [27]. Antiinflammatory effect of thymulin in the brain tissue (production of proinflammatory cytokines) plays role in the maintenance of neurogenesis [28].

Csaba [5] stressed that immunoendocrine thymus is a pacemaker of lifespan and its coinvolution with pineal gland can determine the aging. In the opinion of Mate and co-workers [3], impairment of circadian and seasonal fluctuations of immune functions in human aging may be connected with disturbances of melatonin production. According to our data, the thymic endocrine function in thymulin production is the important part of chronobiological organization of immune system $[19,27]$. The circadian and circannual rhythms of thymulin depend on the synchronizing influence of melatonin. Melatonin production disorders lead to the intra-immune desynchronosis via disturbances of the circadian and circannual rhythms of thymulin and its interactions with the endocrine glands, both in aging and age-related diseases [10,11,19,27,29].

\section{Rhythmicity of Fluctuations in the Pineal Gland and Immune System Functions in Neurodegenerative} Diseases

Parkinson's disease (PD), Alzheimer's disease (AD) and brain ischemia are the wide spread neurodegenerative pathologies [30]. Although multiple sclerosis (MS) is the demyelination disease, today it is also considered as a neurodegenerative disease which 
may occur not only in young subjects but also after age 45 [31]. Progressive neuron loss was shown in neurodegenerative diseases. The pathogenic mechanisms of these diseases include enhancement of oxidative stress, neuroinflammation along with microglia activation, mitochondrial dysfunction, age-related disturbances in the main regions of brain neurogenesis, etc. Disturbances of the sleep-wake cycle and neuroendocrine-immune system functioning during major neurodegenerative diseases may be connected with the pineal gland dysfunction.

\section{Alzheimer's Disease}

Hardeland [32], Skene and Swaab [33] have shown more intensive age-related decrease of the nocturnal blood melatonin level in $\mathrm{AD}$ patients compared to control group that correlates with the development of daily desynchronosis of the blood ACTH and cortisol levels and cognitive dysfunction [9]. It is important that sleep-wake and circadian abnormalities often occur at early stages of $\mathrm{AD}$ and may precede the development of cognitive symptoms [34]. At the same time, melatonin treatment improves sleepwake rhythm and produces antiapoptotic, antioxidant and antiinflammatory effects in the brain in such pathology [32].

AD pathogenesis may be connected with activation of immune cells [35]. Thus, infiltration of T cells and mononuclear phagocytes into injured brain tissues and activation of brain microglia in $\mathrm{AD}$ were clearly shown [36].

\section{Brain Ischemia}

In the elderly patients with acute phase of ischemic stroke the nocturnal urinary melatonin excretion is decreased [37]. In the opinion of Escribano and co-worker [38], decrease of melatonin level in this pathology is a part of its pathogenic features. Thus, in cerebral ischemia melatonin reveals antioxidant, antiapoptotic and anti-inflammatory properties [39]. In ischemic stroke patients the impaired nocturnal melatonin excretion is associated with altered sleep-wake rhythm, increased blood cortisol level and decrease of $\mathrm{T}$ lymphocytes number [40]. In our experimental brain ischemia, the decreasing blood melatonin concentration correlates with the falling of thymulin blood level [19]. Peripheral immune cells may migrate into the site of brain injury and release cytokines and chemokines and, as a result, cause further injury of the ischemic brain [41].

\section{Parkinson's Disease}

PD is characterized by circadian disturbances of melatonin release. Thus, increase of the morning blood melatonin level was shown in patients [42] and suppression of nighttime peak of hormone [43] compared to healthy control group. Such melatonin changes correlate positively with degree of destroy sleep circadian rhythm in PD patients [43]. Also there are circadian abnormalities in blood cortisol release [44]. On the other hand, clinical investigators have shown the slowing of PD development after melatonin treatment that may be connected with antioxidant, antiinflammatory and chronobiological effects of the hormone [45].
Active peripheral immune cells (T lymphocytes, neutrophils, macrophages, etc) play role in the damage of dopaminergic neurons of SCN after their infiltration into the brain $[46,47]$. In our study, the degree of immune disturbances in experimental parkinsonism may be connected with the pronouncement of the decrease of blood thymulin level [48]. The thymic endocrine function was restored after melatonin administration [48].

\section{Multiple Sclerosis}

As shown by Damasceno and co-workers [49], in MS patients the degree of decreasing of night melatonin excretion is a biomarker of disease severity. Such changes of melatonin circadian rhythm are associated with circadian rhythm sleep disturbances [50] and destroy of daily cortisol level rhythmicity [51] in MS patients. The higher frequency of MS attacts in the spring/summer period against winter season is linked with a more marked decrease of nocturnal melatonin level in these seasons [52]. Melatonin treatment improves serum antioxidative properties and life quality of the MS patients [53].

It is known that activation of immune system cells leads to damage of myelin and promotes the development of inflammation in the central nervous system [54]. The pineal gland dysfunction may be pathogenetic factor of such immune disturbances. Thus, in MS patient's melatonin decreases the formation of pathogenic Th17 T cells, cytokine interleukin (IL)-17 and stimulates formation of protective $\operatorname{Tr} 1$ regulatory cells and anti-inflammatory cytokine IL-10 [55]. We have shown that old organism's response at neurodegenerative pathology (multiple sclerosis) to the influence of neurotrophic factors may be changed [56].

Deckx and co-workers [54] stressed that further studies of the neuroendocrine-immune interactions will lead to the development of new therapeutic schemes for treatment of MS patients.

\section{Gender Differences in Neurodegenerative Pathology}

The issue of gender differences in the frequency and symptomatic of neurodegenerative diseases deserves attention [57]. For example more men than women are diagnosed with PD [58]. In the opinion of Miller and Cronin-Golomb [58] these sex differences may be link with female neuroendocrine balance, in particular estrogen action on brain neurons. Moreover, there are data showing that estrogens can modulate rhythmicity of organism functions acting via estrogen receptors in SCN and possess neurotrophic effect [15]. In our data, the women revealed less intensive and slower age-related development of melatonin and thymulin circannual rhythmicity disorders. The latter correlates with less expressive age-related disturbances of the rhythmicity of ACTH and cortisol levels [11].

\section{Conclusion}

The frequency of neurodegenerative diseases increases worldwide. Therefore investigation of new pathogenic factors of their development is worthwhile, in particular the role of pineal 
gland and thymus in the mechanisms of circadian and circannual rhythms disorders of the neuroendocrine and immune systems.

The rhythmicity of immune and neuroendocrine systems functions and their central regulators, melatonin and thymulin, are disturbed in human aging. This process becomes more intensive during development of the main neurodegenerative diseases. Melatonin treatment leads to the suppression of age-related changes in the rhythmicity of immune and neuroendocrine systems and slows the development of neurodegenerative pathology. In the sense of the mechanism of such melatonin effect, we specially stress synchronous influence of this hormone on the circadian and circannual rhythms of blood thymulin.

Therefore melatonin seems to be perspective for restoring disturbed rhythmicity of immune and neuroendocrine systems in the risk groups with accelerated human aging. Also, corrected individual doses of melatonin can be useful for restoration of the rhythmicity of immune-neuroendocrine interactions in patients of different age and sex suffering from neurodegenerative diseases.

In summing up, owing to melatonin properties of improving the rhythms of neuroendocrine and immune systems we recommend melatonin as part of the treatment schemes for patients with other age-related pathologies (osteoporosis, cardiovascular diseases) [19].

\section{References}

1. Reinberg A, Ashkenazi I (2003) Concepts in human biological rhythms. Dialogues Clin Neurosci 5(4): 327-342.

2. Klerman EB (2005) Clinical aspects of human circadian rhythms. J Biol Rhythms 20(4): 375-386.

3. Mate I, Madrid JA, De la Fuerite M (2014) Chronobiology of the neuroimmunoendocrine system and aging. Curr Pharm Des 20(29): $4642-4655$.

4. Reiter RJ, Rosales-Corral S, Coto-Montes A, Boga JA, Tan DX, et al. (2011) The photoperiod, circadian regulation and chronodisruption: requisite interplay between the suprachiasmatic nuclei and the pineal and gut melatonin. J Physiol Pharmacol 62(3): 269-274.

5. Csaba G (2016) The immunoendocrine thymus as a pacemaker of lifespan. Acta Microbiol Immunol Hung 63(2): 139-158.

6. Touitou Y (2001) Human aging and melatonin. Clinical relevance. Exp Gerontol 36(7): 1083-1100.

7. Hirokawa K, Utsuyama M, Makinodan T (2006) Immunity and aging. In: Wiley MSJ (Ed.), Principles and Practice of Geriatric Medicine 2: 19-37.

8. Korkushko OV, Khavinson VKh, Shatilo VB (2006) Pineal gland: ways for correction in aging. St Petersburg, Nauka, Russia.

9. Ferrari E, Arcaini A, Gornati R, Pelanconi L, Cravello L, et al. (2000) Pineal and pituitary-adrenocortical function in physiological aging and in senile dementia. Exp Gerontol 35(9-10): 1239-1250.

10. Labunets IF (2005) Melatonin influence on rhythms of thymus, immune system and adrenal cortex functions in old persons. Probl Aging Longevity 14(4): 313-322.

11. Labunets IF (2013) Sex peculiarities of age-related changes in circannual rhythms of pineal gland, hypothalamo-pituitary-adrenal axis and thymus in healthy subjects. Adv Gerontol 3(4): 290-296.

12. Moriya T, Hiraishi K, Horie N, Mitome M, Shinohara K (2007) Correlative association between circadian expression of mouse Per2 gene and the proliferation of the neural stem cells. Neuroscience 146(2): 494-498.

13. Bellesi M, Pfister-Genskow M, Maret S, Keles S, Tononi G, at al. (2013) Effects of sleep and wake on oligodendrocytes and their precursors. J Neurosci 33(36): 14288-14300.

14. Sotthibundhu A, Phansuwan-Pujito P, Govitrapong P (2010) Melatonin increases proliferation of cultured neural stem cells obtained from adult mouse subventricular zone. J Pineal Res 49(3): 291-300.

15. Vida B, Hrabovszky E, Kalamatianos T, Coen CW, Liposits Z, at al. (2008) Oestrogen receptors $\alpha$ and $\beta$ immunoreactive cells in the suprachiasmatic nucleus of mice: distribution, sex differences and regulation by gonadal hormones. J Neuroendocrinol 20(11): 1270-1277.

16. Numakawa T, Adachi N, Richards M, Chiba S, Kunugi H (2012) The influence of glucocorticoids on neuronal survival and synaptic function. BioMol Concepts 3(6): 495-504.

17. Logan R W, Sarkar DK (2012) Circadian nature of immune function. Mol Cell Endocrinol 349(1): 82-90.

18. Labunets IF, Grinevich YuA (2014) Biological rhythms of immune system functions and possibilities of their regulation in patients with malignant tumours (review of published and authors' own research data). Clin Oncol 2(14): 46-52.

19. Labunets I (2015) Immune-neuroendocrine interactions involving thymus and pineal gland in stem cell therapy of age-related diseases. Immunology, Endocrine \& Metabolic Agents in Medicinal Chemistry (IEMAMC) 15(2): 101-120.

20. Scheirmann Ch, Kunisaki Y, Frenette PS (2013) Circadian control of the immune system. Nat Rev Immunol 13(3): 190-198.

21. Geiger SS, Fagundes CT, Siegel RM (2015) Chrono-immunology:progress and challenges in understanding links between the circadian and immune systems. Immunology 146(3): 349-358.

22. Reggiani PC, Schwerdt Ji, Console GM, Roggero EA, Dardenne M, et al. (2014) Physiology and therapeutic potential of the thymic peptide thymulin. Curr Pharm Des 20(29): 4690-4696.

23. Bach JF, Bach MA, Blanot D, Ericas E, Charreire J, et al. (1978) Thymic serum factor (FTS). Bull Inst Pasteur, Paris, 76: 325-398.

24. Molinero P, Soutto M, Benot S, Hmadcha A, Guerrero JM (2000) Melatonin is responsible for nocturnal increase observed in serum and thymus of thymosin alpha 1 and thymulin concentrations: observations in rats and humans. J Neuroimmunol 103(2): 180-188.

25. Cardinali DP, Esquifino AI, Srinivasan V, Pandi-Perumal SR (2008) Melatonin and the immune system in aging. Neuroimmunomodulation 15(4-6): 272-278.

26. Savino W, Mendes-da-Cruz DA, Lepletier A, Dardenne M (2016) Hormonal control of $\mathrm{T}$ cell development in health and disease. Nat Rev Endocrinol 12(2): 77-89.

27. Labunets I (2012) Pineal gland and rhythms of immune system in aging. Experimental study. LAP LAMBERT Acad Publ: Saarbrucken.

28. Haddad JJ, Hanbali LH (2013) The anti-inflammatory and immunomodulatory activity of thymulin peptide is NF-kB dependent and involves the downregulation of I kB- $\alpha$. Am J Med Biol Res 1(2): 4149.

29. Labunets IF, Rodnichenko AE, Butenko GM (2011) The influence of pineal factors on the bone marrow cell profile in animals of different ages during changes of the thymus functional conditions. Adv Gerontol 1(4): 304-309.

30. Lindvall O, Kokaia Z (2010) Stem cells in human neurodegenerative disorders-time for clinical translation? J Clin Invest 120(1): 29-40.

31. Ismailov MG, Shevchenko PP, Yaschenko IA (2014) Multiple sclerosis and debut in the elderly. Adv Curr Nat Sci 6: 122-123. 
32. Hardeland R (2012) Melatonin in aging and disease-multiple consequences of reduced secretion, options and limits of treatment. Aging Dis 3(2): 194-225.

33. Skene DJ, Swaab DF (2003) Melatonin rhythmicity: effect of age and Alzheimer's desease. Exp Gerontol 38(1-2): 199-206.

34. Musiek ES, Xiong DD, Holtzman DM (2015) Sleep, circadian rhythm, and the pathogenesis of Alzheimer disease. Exp Mol Med (47): e148.

35. McGeer PL, Akiyama H, Itagaki S, McGeer EG (1989) Immune system response in Alzheimer's disease. Can J Neurol Sci 16(4 Suppl): 516-527.

36. Town T (2010) Inflammation, immunity and Alzheimer's disease. CNS Neurol Disord Drug Targets 9(2): 129-131.

37. Ritzenthaler Th, Nighoghossian N, Berthiller J, Schott AM, Cho TH, et al. (2009) Nocturnal urine melatonin and 6-sulphatoxymelatonin excretion at the acute stage of ischaemic stroke. J Pineal Res 46(3): 349-352.

38. Escribano BM, Colin-Gonzalez AL, Santamaria A, Tunez I (2014) The role of melatonin in multiple sclerosis, Huntington's diseases and cerebral ischemia. CNS Neurol Disord Drug Targets 13(6): 1096-1119.

39. Andrabi SS, Parvez S, Tabassum H (2015) Melatonin and Ischemic Stroke: Mechanistic Roles and Action. Adv Pharm Sci 2015: 384750

40. Fiorina P, Lattuada G, Silvestrini C, Ponari O, Dall'Aglio P, et al. (1999) Disruption of nocturnal melatonin rhythm and immunological involvement in ischaemic stroke patients. Scand J Immunol 50(2): 228231.

41. Chengrui A, Yejie Sh, Peiying L, Xiaoming H, Yu G, et al. (2014) Molecular dialogs between the ischemic brain and the peripheral immune system: Dualistic roles in injury and repair. Prog Neurobiol 115: 6-24.

42. Lin L, Du Y, Yuan S, Shen J, Lin X, et al. (2014) Serum melatonin is an alternative index of Parkinson's disease severity. Brain Res 1547: 43-48.

43. Videnovic A, Noble C, Reid K J, Peng J, Turek FW, et al. (2014) Circadian melatonin rhythm and excessive daytime sleepiness in Parkinson disease. JAMA Neurol 71(4): 463-469.

44. Hood S, Amir Sh (2017) Neurodegeneration and the circadian clock. Front in Aging Neurosci 9: p. 170.

45. Srinivasan V, Cardinali DP, Srinivasan US, Kaur C, Brown GM, et al (2011) Therapeutic potential of melatonin and its analogs in Parkinson's disease: focus on sleep and neuroprotection. Ther Adv Neurol Disord 4(5): 297-317.

46. Maguire-Zeiss KA, Federoff HJ (2010) Future directions for immune modulation in neurodegenerative disorders: focus on Parkinson's disease. J Neural Transm 117(8): 1019-1025.
47. Monahan AJ, Warren M, Carvey PM (2008) Neuroinflammation and peripheral immune infiltration in Parkinson's disease: an autoimmune hypothesis. Cell Transplant 17(4): 363-372.

48. Labunets IF, Talanov SA, Vasilyev RG, Rodnichenko AE, Utko NA, et al (2016) Thymic hormones, antioxidant enzymes and neurogenesis in bulbus olfactorius of rats with hemiparkinsonism: effect of melatonin. Int J Physiol Pathophysiol 7(4): 285-298.

49. Damasceno A, Moraes AS, Farias A, Damasceno BP, dos Santos LM, et al (2015) Disruption of melatonin circadian rhythm production is related to multiple sclerosis severity: A preliminary study. J Neurol Sci 353(1-2): 166-168.

50. Najafi MR, Toghianifar N, Etemadifar M, Haghighi S, Maghzi AH, et al. (2013) Circadian rhythm sleep disorders in patients with multiple sclerosis and its association with fatique: a case control study. J Res Med Sci 18(Suppl 1): S71-S73.

51. Kem S, Schultheiss T, Schneider H, SchrempfW, Reichmann H, et al. (2011) Circadian cortisol, depressive symptoms and neurogical impairment i early multiple sclerosis. Psychoneuroendocrinology 36(10): 1505-1512.

52. Wurtman R (2017) Multiple sclerosis, melatonin and neurobehavioral diseases. Front Endocrinol.

53. Adamczyk-Sowa M, Pierzchala K, Sowa P, Polaniak R, Kukla M, et al. (2014) Influence of melatonin supplementation on serum antioxidative properties and impact of the quality of life in multiple sclerosis patients. J Physiol Pharmacol 65(4): 543-550.

54. Deckx N, Wai-Ping Lee, Berneman ZN, Cools N (2013) Neuroendocrine immunoregulation in multiple sclerosis. Clin Dev Immunol 2013: 705232 .

55. Farez MF, Mascancroni ID, Mendez-Huergo SP, Rabinovich GA, Quintana FJ, et al. (2015) Melatonin contributes to the seasonality of multiple sclerosis relapses. Cell 162(6): 1338-1352.

56. Labunets IF, Melnyk NO, Rodnichenko AE, Rymar SE, Utko NA (2017) Cuprizone-Induced Disorders of Central Nervous System Neurons, Behavioral Reactions, Brain Activity of Macrophages and Antioxidant Enzymes in the Mice of Different Ages: Role of Leukemia Inhibitory Factor in their Improvement. J Aging Geriatr Med 1(2): pp. 1-8.

57. Zagni E, Simoni L, Colombo D (2016) Sex and gender differences in central nervous system-related disorders. Neurosci J 2016: 2827090.

58. Miller IN, Cronin-Golomb A (2010) Gender differences in Parkinson's diseases: clinical characteristics and cognition. Mov Disord 25(16): 2695-2703. 